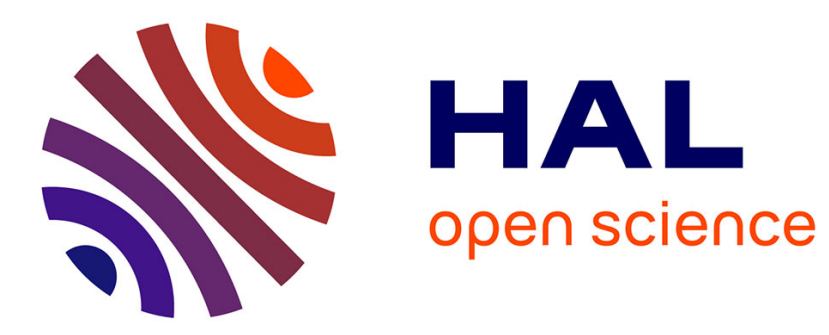

\title{
First Order Bifurcation Landscape in a 2D Geometry: The Example of Solid Friction
}

\author{
Arnaud Tanguy, Ph. Nozières
}

\section{To cite this version:}

Arnaud Tanguy, Ph. Nozières. First Order Bifurcation Landscape in a 2D Geometry: The Example of Solid Friction. Journal de Physique I, 1996, 6 (10), pp.1251-1270. 10.1051/jp1:1996136 . jpa00247244

\section{HAL Id: jpa-00247244 https://hal.science/jpa-00247244}

Submitted on 1 Jan 1996

HAL is a multi-disciplinary open access archive for the deposit and dissemination of scientific research documents, whether they are published or not. The documents may come from teaching and research institutions in France or abroad, or from public or private research centers.
L'archive ouverte pluridisciplinaire HAL, est destinée au dépôt et à la diffusion de documents scientifiques de niveau recherche, publiés ou non, émanant des établissements d'enseignement et de recherche français ou étrangers, des laboratoires publics ou privés. 


\title{
First Order Bifurcation Landscape in a 2D Geometry: The Example of Solid Friction
}

\author{
A. Tanguy $(1, *)$ and $\mathrm{Ph}$. Nozières $\left({ }^{2}\right)$ \\ (1) LPMMH, ESPCI, 10 rue Vauquelin, 75005 Paris, France \\ $\left({ }^{2}\right)$ Institut Laue Langevin, BP. 156X, 38042 Grenoble Cedex 9, France
}

(Recelved 3 May 1996, received in final form 17 June 1996, accepted 27 June 1996)

PACS.05.70Fh - Phase transitions: general aspects

\begin{abstract}
The generalization to $2 \mathrm{D}$ of a model for quassstatic friction reveals the importance of anisotropy in the pinning potential between the asperities on slider and track. This ansotropy, which breaks the rotational symmetry of the potential, changes, qualitatively as well as quantitatively, the dissipative behaviour of the asperity. This study alms to show, with the help of an example, the unfolding of bifurcation diagrams for first order transitions when the state space is changed from one to two dimensions.
\end{abstract}

Résumé. - La généralisation à $2 \mathrm{D}$ d'un modèle de frottement quasistatique met en évıdence l'importance de l'anisotropie du potentiel d'accrochage entre une aspérité du patin et une aspérité de la piste. Cette anısotropıe, qu brise la symétrie de rotation du potentiel, change qualitativement et quantitativement le comportement dissipatif de l'aspérité. Cette étude précise à l'aide d'un exemple le déploiement du diagramme de bifurcation des transitions du premier ordre lorsque l'espace des états passe de une à deux dimensions.

\section{Introduction}

Reference [1] describes a model for solid friction based on a hysteretic elastic mechanism which involves the asperities at the surfaces of two solids in contact. This model is very simple in the case of a single contact. The contact is characterized by a nominal coordinate $\rho$, which measures the relative positions of asperities facing each other, on the track and slider respectively, in the absence of any elastic response. When the slider moves, $\rho$ is swept from $-\infty$ to $+\infty$. Due to the pinning potential acting on the contact, both slider and track undergo elastic deformation and the relative coordinate moves from $\rho$ to $r=\rho+u$. The total potential energy of the asperity may be written as

$$
U=V(r)+\frac{\lambda}{2}(r-\rho)^{2}
$$

where $V(r)$ is the pinnung potential and $\lambda$ is an elastic coefficient. To a first approximation, we neglect the interaction between contacts, so we can keep $\rho$ as a control parameter. If $\lambda$ is small enough ("soft" system), the equlibrium position $r$ has a range of multistability, leading to a hysteretic regime responsible for the dissipation in the quasistatic limit. (During

$\left({ }^{*}\right)$ Author for correspondence (e-mall: tanguy@manet.pmmh.espcu.fr) 


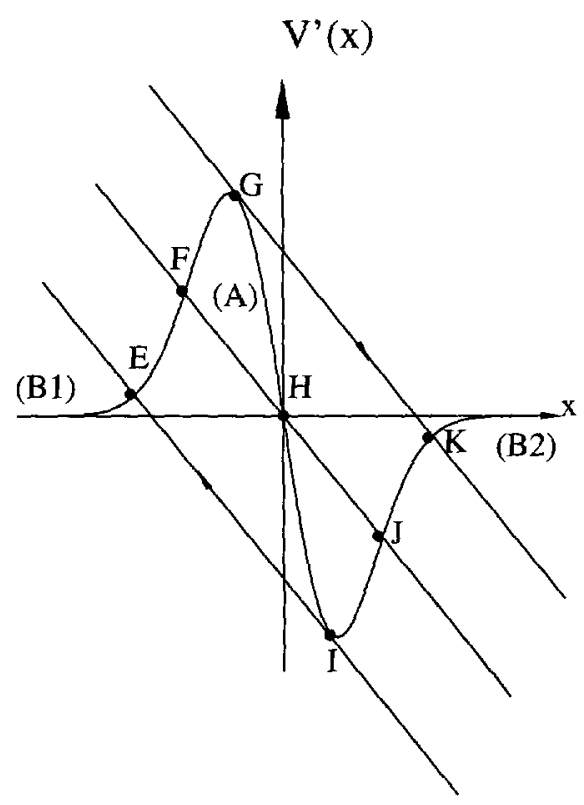

Fig. 1. - Graphical construction of the equilibrium positions in the ID case. $V^{\prime}(x)$ is cut by a straight line of slope $(-\lambda)$ intersecting the $x$-axis at $\rho$. GK and IE are the hysteretic jumps, FJ is the Maxwell plateau.

the discontinuous jump from one stable equilibrium state to another, a bunch of phonons is radiated into the solid, leading to irreversible behavıor.)

The analysis given in [1] is one-dimensional: it leads to the classical bifurcation scheme of a first order transition [2]. The aim of this paper is to generalize the discussion to the 2D case. Independently of the example of friction, the issue is to generalize the "bifurcation landscape" from a 1D system to a 2D system. This is a question of general interest, whose answer is not so obvious. We shall attempt to give a qualitative answer. For the sake of simplification, we first discuss the case of a repulsive pinning potential. After reviewing the 1D-case, we discuss the case of an isotropic 2D-potential in which the hysteresis disappears. We show that the anisotropy plays a determinant role in the restoration of multistability. We thus obtain a coherent picture of the bifurcation landscape. The case of an attractive potential is briefly discussed in Section 4.

\section{Presentation of the quasi 1D-Model}

The behaviour of a repulsive potential as a function of $x$ is sketched in Figure 2. We can take, if necessary, a Gaussian potential

$$
V(x)=V \mathrm{e}^{-\frac{x^{2}}{2 a^{2}}}
$$

that meets the condition of no interaction at infinity. For a given nominal position $\rho_{x}=\rho$, the elastic displacement adjusts itself in such a way'as to minimize the potential energy $U$ at equilibrium according to:

$$
\frac{\mathrm{d} U}{\mathrm{~d} x}=\frac{\mathrm{d} V(x)}{\mathrm{d} x}+\lambda(x-\rho(t))=0
$$




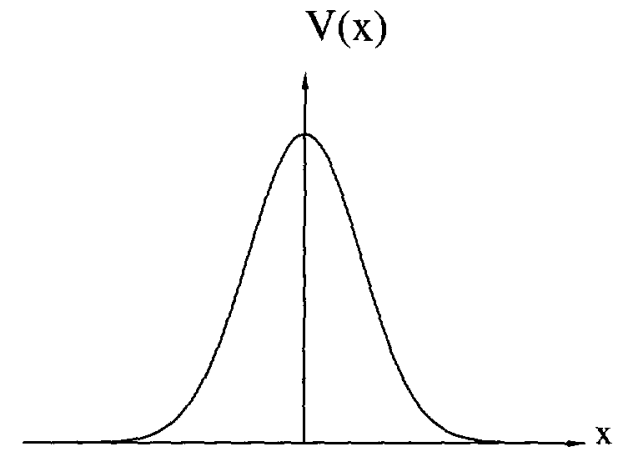

Fig. 2. - Repulsive pınnıng potential.

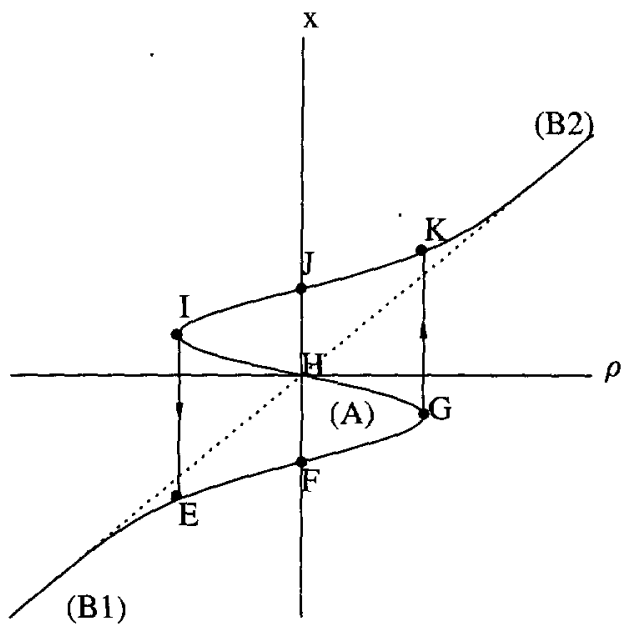

Fig. 3. - Behaviour of the equlibrium positions $x$ as a function of the control parameter $\rho$ (catastrophe manfold).

The equilibrium is stable if

$$
\frac{\mathrm{d}^{2} U}{\mathrm{~d} x^{2}}=\frac{\mathrm{d}^{2} V}{\mathrm{~d} x^{2}}+\lambda>0
$$

Equation (3) leads itself to a graphical solution, as illustrated in Figure 1: $V^{-\prime}(x)$ is cut by a straight line of slope $(-\lambda)$ intersecting the $x$-axis at $\rho$. Two cases may occur:

- If min $V^{\prime \prime}+\lambda>0$, each nominal position $\rho$ corresponds to a single equilibrium position $x$ : this monostable system is called "hard". Such a system does not dissipate energy.

- If $\min V^{\prime \prime}+\lambda<0$, the system is "soft": it generates a zone of multistability. The behavior of $x$ as a function of $\rho$ is illustrated in Figure 3. Here, G and I are the spinodal limits where the metastable solution annihilates with the unstable solution. The region GI is unstable. Points in the stable regions occurrıng before $G$ and after $I$ are referred to as $\left(B_{1}\right)$ and $\left(B_{2}\right)$ respectively, while a point in the unstable region GI is labelled $(A)$.

The total energy $U_{\mathrm{eff}}(\rho)$ is illustrated in Figure 4, where the characteristic points of Figure 3 are shown. The double point FJ corresponds to the Maxwell plateau. At this 
point, the two metastable states $\left(B_{1}\right)$ and $\left(B_{2}\right)$ have the same energy. Beyond this point $\left(B_{1}\right)$ is no longer a global energy minimum. If the asperity could jump over the barrier (A) by thermal activation, then the jump from one branch to the other would occur at this location. In a first approximation, this jump is forbidden. The system is governed by the "delay rule" [3]. When $\rho$ is increasing from $-\infty$, the slider travels up to $G$ where the asperity jumps on to the other branch at K. During the return journey, the slider goes to I before the asperity jumps. The behavior of the system is hysteretic and irreversible.

\section{Quasi-2D Isotropic Repulsive Model}

The pinning potential (Fig. 2) is rotationnaly symmetric. The real position $\mathbf{r}$ and the nominal position $\rho(t)$ are now vectors. When sweeping along the $x$-direction, the component $\rho_{y}(t)$ acts as a constant impact parameter which we control. The equations of motion, expressed in polar coordinates in $r$-space, are:

$$
\begin{gathered}
\frac{\mathrm{d} V}{\mathrm{~d} r}+\lambda(\mathbf{r}-\boldsymbol{\rho}) \cdot \mathbf{u}_{r}=0 \\
0+\lambda(\mathbf{r}-\boldsymbol{\rho}) \cdot \mathbf{u}_{\theta}=0
\end{gathered}
$$

The jumps effected by the asperity can only be radial jumps ( $\mathbf{r}-\boldsymbol{\rho}$ is radial). The effective energy is thus rotationally symmetric in the parameter space described by

$$
\left(\rho_{x}, \rho_{y}\right)=(\rho \cos \theta, \rho \sin \theta)
$$

The shape of the effective energy (Fig. 4) is simply obtained by rotating the 1D-curve of Figure 4 around the vertical axis.

The stability of equilibrium positions is now given by the sign of the eigenvalues of the Hessian matrix $\mathrm{d}^{2} U / \mathrm{du}^{2}$, which in this case are

$$
\left(V^{\prime \prime}(r)+\lambda\right) \text { and }\left(\frac{V^{\prime}(r)}{r}+\lambda\right)
$$

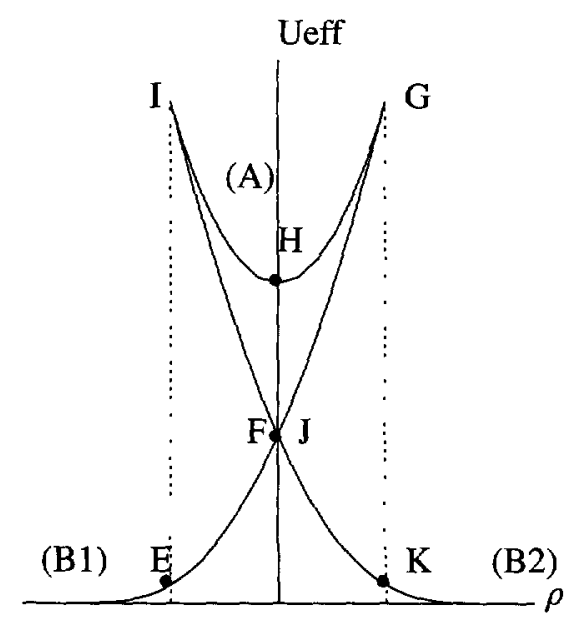

Fig. 4. - Effective energy (energy of the equllbrium positions) as a function of $\rho$. The upper branch IHG is unstable. 


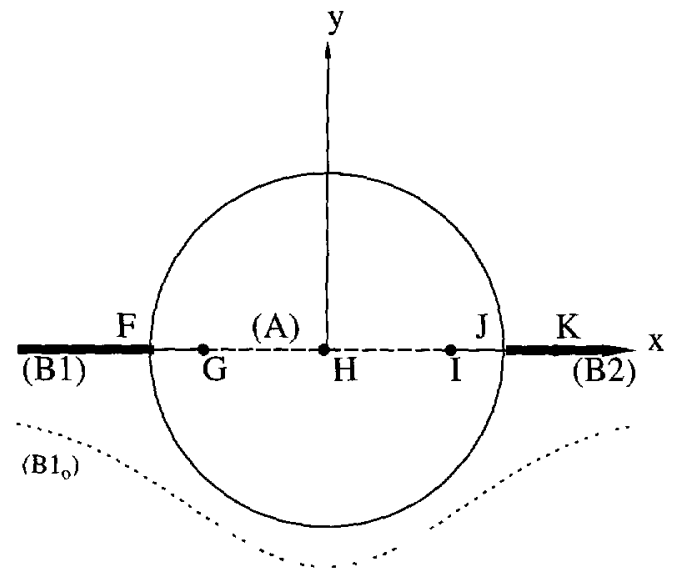

Fig. 5. - Equilibrium positions in the $(x, y)$ plane for an isotropic $2 \mathrm{D}$ pinning center. On the symmetry axis the thick lines are energy minuma, the central dashed line GI is an energy maximum, FG and IJ are saddle points. The circle is a separatrix, corresponding to equilibrium positions when $\rho_{x}=\rho_{y}=0$. When $\rho_{y} \neq 0$, the trajectory follows the dotted line, with no discontinuity.

These eigenvalues characterize the stability of the system in radial and orthoradial directions respectively. With the symmetric potential of Figure 2, the minima of $V^{\prime \prime}(r)$ and $V^{\prime}(r) / r$ coincide: we retrieve the previous "hard case" and "soft case" threshold condition. In Figure 3, the radial instability occurs on the same region (GI) as in the $1 \mathrm{D}$ case, but the tangential instability occurs over a larger domain, which is limited by the Maxwell plateau (FJ), i.e. by the conic point of $U_{\text {eff }}$. In Figure 4, the upper surface of the cone corresponds to saddle points of $U$, radially stable, but unstable tangentially. The upper surface of the potential energy $U_{\text {eff }}$ is completely unstable.

We can again follow the equilibrium positions $\mathbf{r}$ of the asperity, when $\rho_{x}$ is varied at a constant $\rho_{y}$. Consider first the case $\rho_{y}=0$. A trivial solution is $y=0$ (Eq. (6) is automatically satisfied). We recover the $1 \mathrm{D}$ situation: the equilibrium positions remain along the $x$-axis which is an axis of symmetry for our system. The situation is illustrated in Figure 5, where the labelling is the same as in Figure 3. The segments FG and IJ are saddle points for the potential $U$, GI are maxima. If $\rho=0$, another solution corresponds to $\left(V^{\prime}(r) / r\right)+\lambda=0$, i.e. to a circle of radius $x_{\mathrm{F}}$ corresponding to an arbitrary orientation of the Maxwell plateau. If we did not consider the transverse instability, varying $\rho_{x}$ from left to right would give the same behavior as in the 1D case. The jump would then occur at $\mathrm{G}$ and the asperity would go to $\mathrm{K}$. However, as soon as $\rho_{y} \neq 0$, the asperity follows the dotted trajectory in Figure 5: it avoids point $\mathrm{F}$. The asperity goes around the conic point and the jump disappears. The evolution of $\mathbf{r}$ is continuous and it is no longer dissipative.

We conclude that the tangential anstability destroys the hysteresis. This result is due to the assumption of isotropy. We now study the anisotropic case, in which the disappearance of the hysteresis is more subtle.

\section{Quasi-2D Anisotropic Repulsive Model}

3.1. InTRODUCTION. -- The introduction of anisotropy breaks the rotational symmetry of the effective potential and introduces a new control parameter into our system. By means of 
a change of scale, and assuming decorrelation between the elastic properties and the pinning, we can introduce anisotropy into the elastic stiffness of the system instead of the range of the potential. The generalization of the potential energy of the equation (1) to the anisotropic case is thus given by:

$$
U=V(r)+\frac{\lambda_{x}}{2}\left(x-\rho_{x}(t)\right)^{2}+\frac{\lambda_{y}}{2}\left(y-\rho_{y}(t)\right)^{2}
$$

where $\lambda_{y}<\lambda_{x}$. The anisotropy is characterized by the dimensionless parameter $\lambda_{y} / \lambda_{x}$ which is restricted between 0 and 1 . Note that in comparison with a model which introduces the anisotropy in the range of the pinning potential, a softer stiffness in the $x$-direction corresponds to a smaller range of the pinning potential in this same direction.

3.2. Equilibrium Positions. - Equilibrium positions correspond to an extremum of $U$, i.e. to

$$
\begin{aligned}
& x\left(\lambda_{x}+\frac{V^{\prime}(r)}{r}\right)=\lambda_{x} \rho_{x}(t) \\
& y\left(\lambda_{y}+\frac{V^{\prime}(r)}{r}\right)=\lambda_{y} \rho_{y}(t)
\end{aligned}
$$

(where $V^{\prime}(r)=\partial V / \partial r$ is the radial derivative). Symmetry is lost except on the $x$ and $y$ axis. Consider for instance the $\rho_{x}$ axis, on which $\rho_{y}=0$ : there two types of solutions may coexist.

(i) Either $y=0$, thereby preserving the $(y \rightarrow-y)$ parity. Then the first equation (8) relates $x$ to $\rho_{x}$ : we recover the $1 \mathrm{D}$ situation, multistability occurs if $\min V^{\prime \prime}+\lambda_{x}<0$.

(ii) Or $\lambda_{y}+\left(V^{\prime}(r) / r\right)=0$, which yields $r$. Combined with the first equation (8), that condition implies

$$
x=\rho_{x} \frac{\lambda_{x}}{\lambda_{x}-\lambda_{y}}
$$

Such solutions exist if $r>x$, with a finite value $\pm y$ : they break the symmetry.

The bifurcation corresponds to $r=x=x^{*}$, i.e. to $\lambda_{y}+\left(V^{\prime}\left(x^{*}\right) / x^{*}\right)=0$. It is of the usual pitchfork type, corresponding to a tangential instability of the symmetric solution when $\lambda_{y}+\left(V^{\prime}(x) / x\right)<0$. Note that the symmetry breaking solution corresponds to $r=x^{*}$ : using (9) the value of $|y|$ follows.

In order to understand the nature of that bifurcation in more detail, we must follow the evolution of the various roots as the control parameter $\rho_{x}$ is varied away from the bifurcation $\rho_{x}^{*}$ : what matters is the sign of $\mathrm{d} x / \mathrm{d} \rho_{x}$. For the symmetric solution $y=0, x=x_{0}$, it follows from (8) that

$$
\frac{\mathrm{d} x_{0}}{\mathrm{~d} \rho_{x}}=\frac{\lambda_{x}}{\lambda_{x}+V^{\prime \prime}}
$$

As we shall see in the next section that ratio is positive if the solution is radially stable: we only consider that case. $x_{0}$ turns tangentially unstable if $x_{0}<x^{*}, \rho_{x}<\rho_{x}^{*}$. In contrast the symmetry breaking solution $x_{ \pm}$obey (9) and therefore

$$
\frac{\mathrm{d} x_{ \pm}}{\mathrm{d} \rho_{x}}=\frac{\lambda_{x}}{\lambda_{x}-\lambda_{y}}
$$

Since we assumed $\lambda_{y}<\lambda_{x}$ that ratio is also positive: it follows that the bifurcation on the $x$ axis is direct. The two symmetry breaking solutions appear when $\rho_{x}<\rho_{x}^{*}$, on the same s1de of the bifurcation as the unstable branch $x_{0}$, as shown in Figure 6a. 


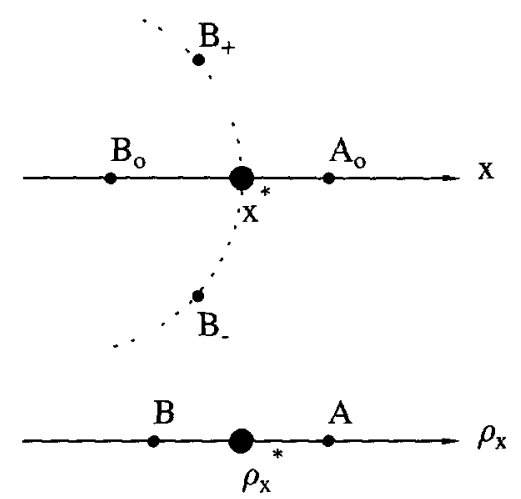

(a)

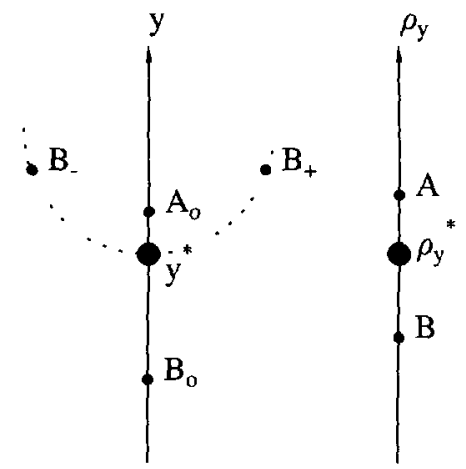

(b)

Fig. 6. - The nature of transverse bifurcation on symmetry axes in the radially stable case. Dark points correspond to the bifurcation. $\mathrm{A}(\mathrm{B})$ in the $\rho$ axis are mapped onto extrema in the $(x, y)$ plane. In both cases, $\mathrm{A}$ are points before the transverse bifurcation and $\mathrm{B}$ are points after the transverse bifurcation for a sweeping in the decreasing $\rho$-direction. On the $x$ axis (Fig. 6a), the bifurcation is direct $\left(A_{0}, B \pm\right.$ are minima, $B_{0}$ is a saddle point). On the $y$ axis (Fig. 6b), the bifurcation is reversed ( $A_{0}, B \pm$ are saddle points, $B_{0}$ is a minımum).

The situation is reversed if we study the $y$ axis, $\rho_{x}=0$, instead of the $x$ axis. Then we must interchange $x$ and $y$ in $(10,11)$. The bifurcation occurs at some $y^{*}, \rho_{y}^{*}$, below which the $x=0$ solution is tangentially unstable. The ratio $\mathrm{d} y / \mathrm{d} \rho_{y}$ remains positive for the solution $y_{0}$, while it is negative for the two symmetry breaking solutions $y_{ \pm}$, corresponding to

$$
r_{ \pm}=y^{*} \quad, \quad y_{ \pm}=\frac{\lambda_{y}}{\lambda_{y}-\lambda_{x}} \rho_{y}
$$

It follows that the bifurcation is inverted. Symmetry breaking solutions appear for values $\rho_{y}>\rho_{y}^{*}$ for which the symmetry axis is transversally stable, as shown in Figure $6 \mathrm{~b}$. While in the case $6 \mathrm{a}$ the bifurcation goes from one minimum to a saddle point surrounded by two minima, in case $6 \mathrm{~b}$ one goes from a minimum surrounded by two saddle points $\left(\rho_{y}>\rho_{y}^{*}\right)$ to a single saddle point $\left(\rho_{y}<\rho_{y}^{*}\right)$. The lateral extrema turn around the origin as they leave the bifurcation $y^{*}$; they eventually merge at the symmetric bifurcation $-y^{*}$. (Technically the transverse stability is reversed by the off diagonal elements of the stability matrix, proportional to $x)$.

Away from the symmetry axis a generic bifurcation corresponds to coalescence of a node with a saddle point. In the direct case the evolution is continuous (in close analogy with a ferromagnet in a finite external field). In the reverse case, in contrast, the only local minimum disappears: a discontinuous jump is unavoidable. That jump persists when $\rho_{x}=0$. Note that it cannot lead to the above saddle points: despite the fact that symmetry breaking solutions exist, the jump is not symmetry breaking. It necessarily ends on the $y$ axis, at a point $y_{\mathrm{f}}$ such that

$$
V^{\prime}\left(y_{\mathrm{f}}\right)=\lambda_{y}\left(\rho_{y}^{*}-y_{\mathrm{f}}\right)
$$

The graphical solution is the same as in $1 \mathrm{D}$ (Fig. 4). This feature is crucial in understanding hysteresis. 
3.3. STABILITY ANALYSIS. - The nature of bifurcations is controlled by local stability of the equilibrium solutions, i.e. by the eigenvalues of the Hessian matrix

$$
H=\left(\begin{array}{cc}
\lambda_{x}+V_{x x}^{\prime \prime} & V_{x y}^{\prime \prime} \\
V_{x y}^{\prime \prime} & \lambda_{y}+V_{y y}^{\prime \prime}
\end{array}\right)
$$

Bifurcations occur when one of the elgenvalues vanishes, i.e. when the determinant $\Delta$ of that matrix is zero. The eigenvector of $H$ with zero elgenvalue defines the soft mode which changes stability. Noting that

$$
V_{x x}^{\prime \prime}=V^{\prime \prime} \frac{x^{2}}{r^{2}}+\frac{V^{\prime}}{r} \frac{y^{2}}{r^{2}} \quad, \quad V_{x y}^{\prime \prime}=\left(V^{\prime \prime}-\frac{V^{\prime}}{r}\right) \frac{x y}{r^{2}}
$$

we easily calculate the determinant $\Delta$

$$
\Delta=V^{\prime \prime} \frac{V^{\prime}}{r}+V^{\prime \prime}\left(\lambda_{y} \cos ^{2} \theta+\lambda_{x} \sin ^{2} \theta\right)+\frac{I^{\prime \prime}}{r}\left(\left(\lambda_{x} \cos ^{2} \theta+\lambda_{y} \sin ^{2} \theta\right)+\lambda_{x} \lambda_{y}\right.
$$

(when $\theta$ is the polar angle in the $(x, y)$ plane). The roots of (14) separate regions of different stabilities.

Along each of the symmetry axes, equation (14) factorizes with decoupled radial and tangential instabilities. The corresponding equations are:

\begin{tabular}{|l|l|l|}
\hline & $x$ axis & $y$ axis \\
\hline radial & $V^{\prime \prime}+\lambda_{x}=0$ & $V^{\prime \prime}+\lambda_{y}=0$ \\
\hline tangential & $\frac{V^{\prime}}{r}+\lambda_{y}=0$ & $\frac{V^{\prime}}{r}+\lambda_{x}=0$ \\
\hline
\end{tabular}

The solution of (15) is shown graphically in Figure 7 . We note that:

(i) Bifurcations only occur for soft systems in which at least the smallest stiffness $\lambda_{y}$ is such that

$$
V_{0}^{\prime \prime}+\lambda_{y}<0
$$

In which $V_{0}^{\prime \prime}$ is the common minimum of $V^{\prime \prime}$ and $V^{\prime} / r$ at $r=0$.

(ii) Since we assumed $\lambda_{y}<\lambda_{x}, \mathrm{~A}_{x}$ (Fig. 7) is always closer to the origin than $\mathrm{B}_{x}$ : the tangential instability occurs first on the $x$-axis. In contrast, the relative positions of $\mathrm{A}_{y}$ and $\mathrm{B}_{y}$ depends on the anisotropy.

This situation is summarized on the phase diagram of Figure 8, which displays four regimes:

- In region I, there is no instabulity at all: only one equilibrium position $r$ exists whatever $\rho$.

- Region II only has one instability, which evolves continuously from a radial mode, on the $y$-axis, to a tangential mode, on the $x$-axis. In between the soft mode has no particular symmetry.

- In region III, a second instability appears closer to the origin $\mathbf{r}=0$. The corresponding soft mode evolves from tangential on the $y$-axis to radial in the $x$-axis. 


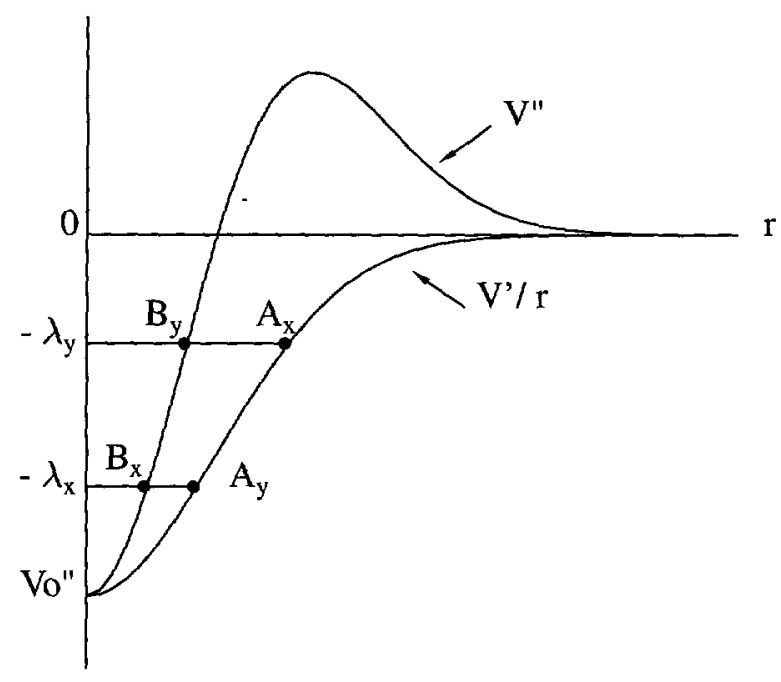

Fig. 7. - Graphical solution of (11). $\mathrm{B}_{x}$ and $\mathrm{B}_{y}$ are radial instabilities respectively on the $x$ and $y$ axes, $\mathrm{A}_{x}$ and $\mathrm{A}_{y}$ are tangential instabilities.

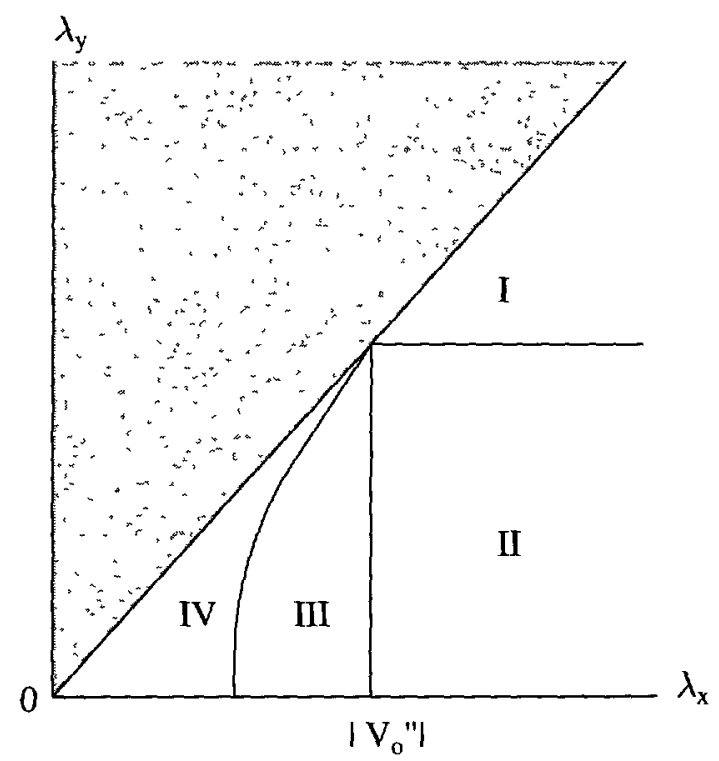

Fig. 8. - Phase diagram displaying the various regimes as a function of $\lambda_{x}$ and $\lambda_{y}$.

- In region IV, the roots along the $y$-axis have crossed. Since the roots of (14) cannot cross for intermediate angles $\theta$ (in the absence of symmetry they repel), that means an interchange of eigenvectors as one moves from $x$ to $y$. The outer bifurcation curve in the $(x-y)$ plane is a strictly tangential instability on the two axis and primarily so in between. The inner curve is primarly radial.

The various situations are displayed in Figure 9, which shows the locus of bifurcations in the $(x, y)$ plane for the three cases II, III, IV. 
y

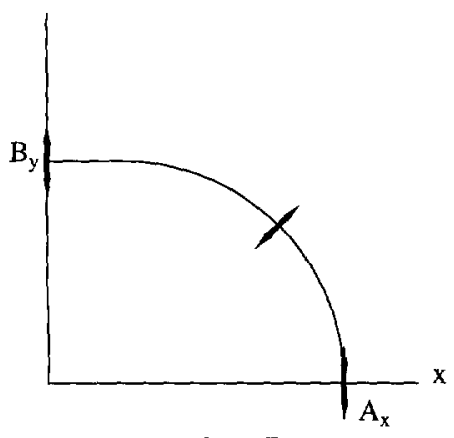

Case II

$$
\mathrm{y}
$$

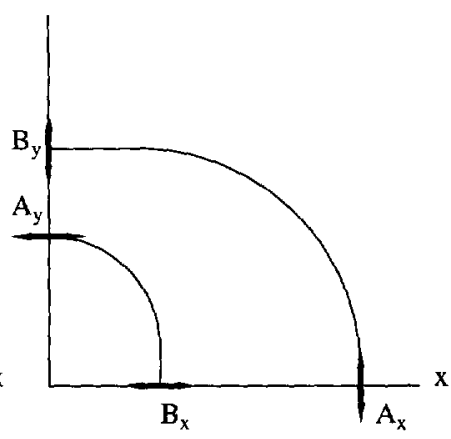

Case III y

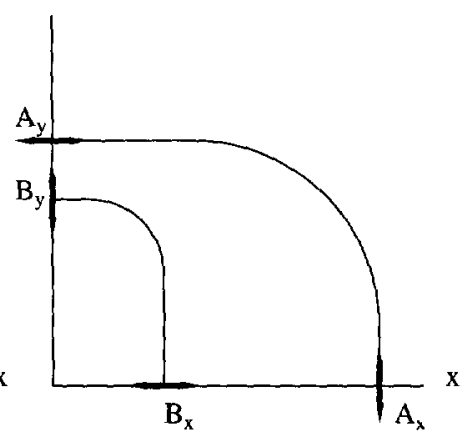

Case IV

Fig. 9. - Locus of bifurcations in the $(x, y)$ plane. The labelling of points is the same as in Figure 6. The arrows denote the polarization of the soft mode at the bifurcation.

Knowing the positions of bifurcations in the $(x, y)$ plane, we can infer the configurational coordinates $\rho$ from (8), thereby constructing the bifurcation diagram in the $\left(\rho_{x}, \rho_{y}\right)$ plane. The latter diagram is the one we need, since $\rho$ is the physical coordinate which is controlled from outside. Detailed results depend on the shape of $V(r)$, but qualitative features can be easily found using a Taylor expansion of $V^{\prime}$ and $V^{\prime \prime}$ :

$$
\begin{aligned}
\frac{V^{\prime}(r)}{r} & =V_{0}^{\prime \prime}\left(1-\alpha r^{2}\right) \\
V^{\prime \prime}(r) & =V_{0}^{\prime \prime}\left(1-3 \alpha r^{2}\right)
\end{aligned}
$$

(Anyhow (16) is valid near $r=0$ ). Expansions near the symmetry axes are straightforward, but somewhat tedious: we sketch them in the appendix and here we give only the results shown in Figure 10. These results call for a number of comments:

(i) The thresholds $\mathrm{A}_{x}, \mathrm{~A}_{y}$ for tangential instabilities on the symmetry axes are imaged into cusps in the $\left(\rho_{x}, \rho_{y}\right)$ plane (see the appendix for precise results). This is the expected behaviour for pitchfork bifurcations. In contrast radial saddle point bifurcations, $\mathrm{B}_{x}, \mathrm{~B}_{y}$, are imaged into regular analytic points in the $\left(\rho_{x}, \rho_{y}\right)$ plane.

(11) Continuity arguments provide a simple description of multistability. Consider for instance case II and assume that $\rho_{y}$ is decreased from $+\infty$, say at $\rho_{x}=0$. The stable solution persist up to $\mathrm{B}_{y}$ in the $(x, y)$ plane, i.e. up to a negative value of $\rho_{y}$. Had we started from $\rho_{y}=-\infty$, we. would proceed to the opposite value of $\rho_{y}$ before meeting instability. It follows that the shaded area of Figure 10 is multistable, with two stable sheets and necessarily one unstable sheet in order to connect them. The total energy $U(\rho)$ has two spinodal limits that merge at the cusps $\mathrm{A}_{x}$, and in between a double line (here the $\rho_{x}$ axis by symmetry) that corresponds to the Maxwell plateau. The same situation repeats a second time in case III: another fold develops, limited by the cusps $A_{y}$. In that case, this secondary fold develops on the unstable sheet of the primary bifurcation: it is physically irrelevant.

(iii) In case IVa, the bifurcation curves cross in the $\left(\rho_{x}, \rho_{y}\right)$ plane, which may look surprising. Such an intersection would be impossible in the $(x, y)$ plane, where there is no multistability (according to (8), $\rho_{x}$ and $\rho_{y}$ are uniquely defined given $x$ and $y$ ). But in the $\left(\rho_{x}, \rho_{y}\right)$ plane the two threshold curves of Figure 10 correspond to different sheets of the equilibrium states: 


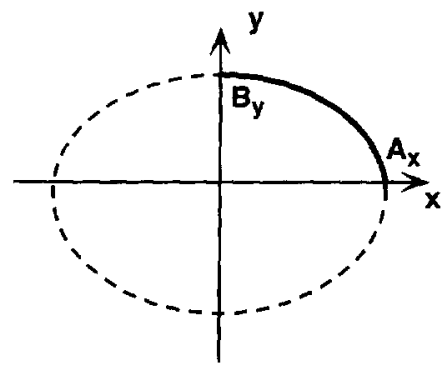

II
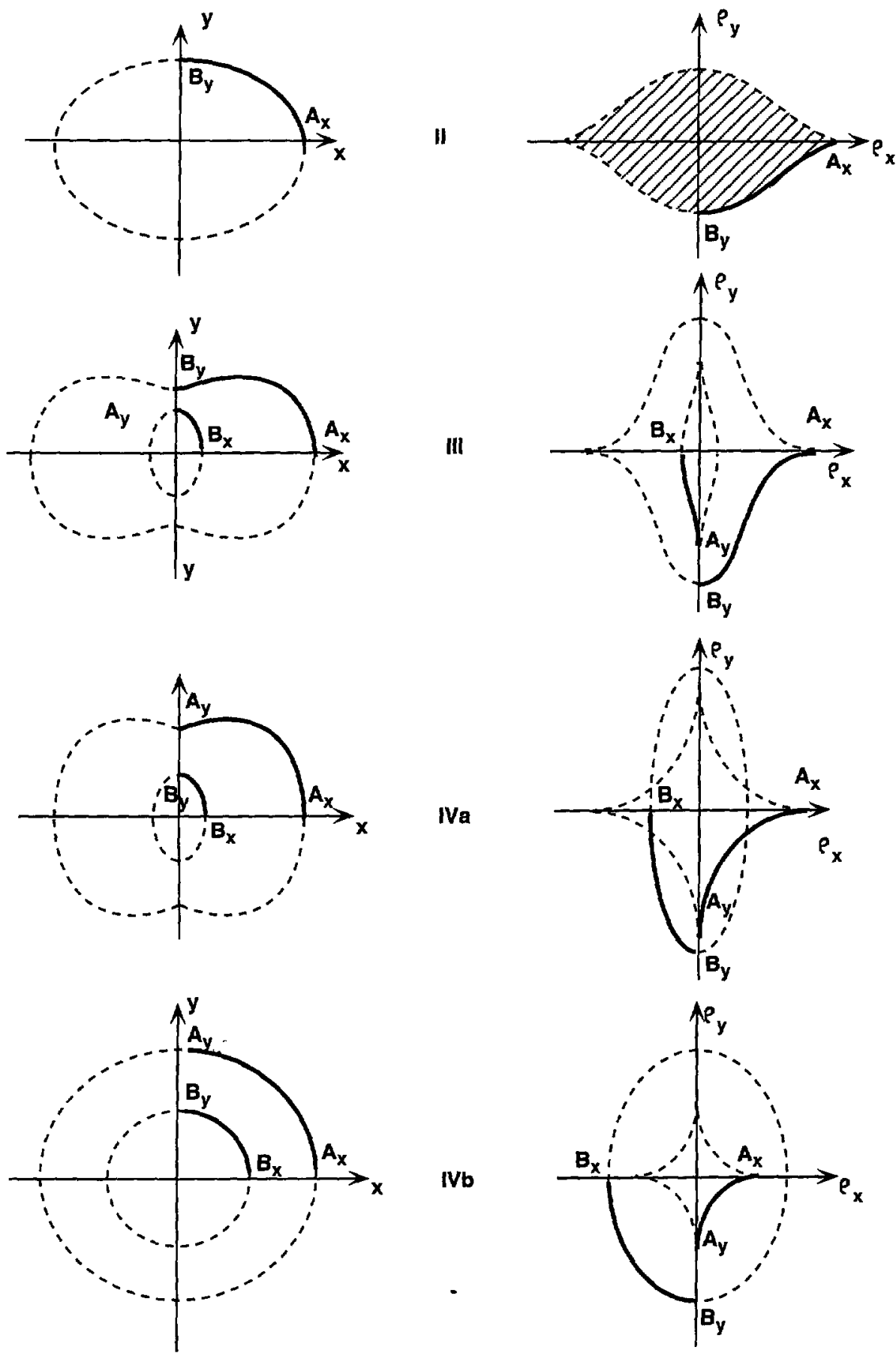

Fig. 10. - Locus of the bifurcations in the $(x, y)$ and $\left(\rho_{x}, \rho_{y}\right)$ planes for the various cases of Figure 8. For each case the labelling of symmetry points is the same in the two figures - and also the same as in Figures 7 and 9. As a guide for the eye, one quadrant of the $(x, y)$ plot is shown in full lines, and the 1mage of that branch in the $\left(\rho_{x}, \rho_{y}\right)$ plot is also shown in full lines: in that way one can follow continuously the evolution of the various sheets in the $\left(\rho_{x}, \rho_{y}\right)$ plane. 


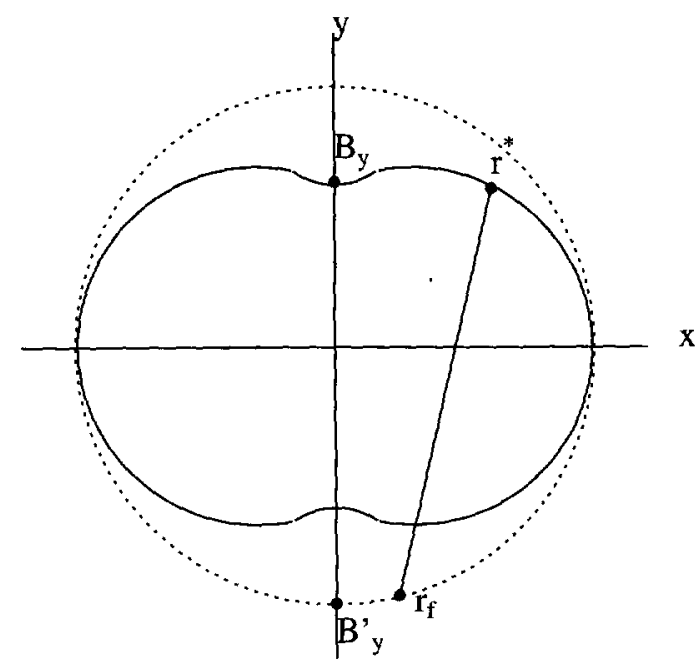

(a)

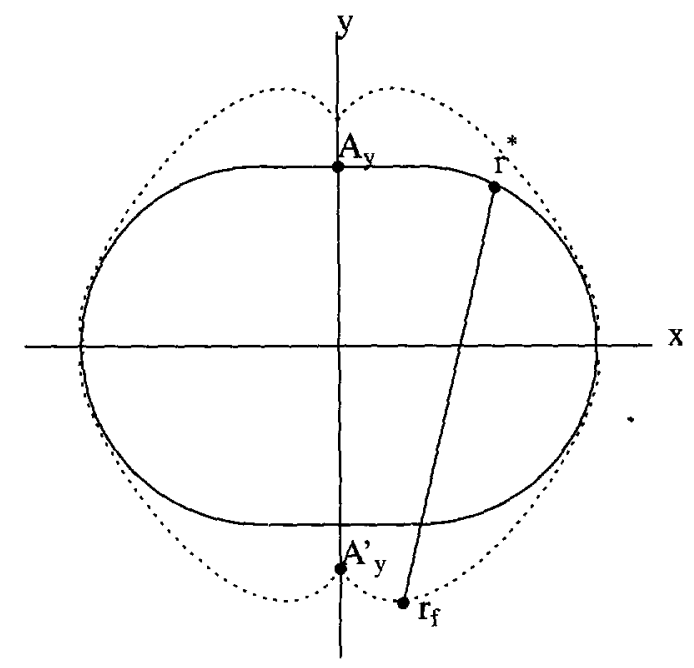

(b)

Fig. 11. - The distribution of discontinuous jumps. Figure 11a corresponds to the cases II or III of Figure 10. Figure 11b corresponds to the case IV of Figure 10. The full curve is the spinodal limit of Figure 9 and the dotted curve the corresponding final position.

there is no repulsion constraint and the bifurcation curves ignore each other: they may well cross. We also note that $\mathrm{A}_{y}$ and $\mathrm{B}_{y}$ do not exchange along the $\rho_{y}$ axis, while they do in the $(x, y)$ plane. We show in the appendix that they approach each other an second order as the boundary of regions III and IV is approached: they concide at the boundary but they do not intersect.

(iv) The case IVb corresponds to very small anisotropies, $\lambda_{y}$ and $\lambda_{x}$ being very close. In the isotropic limit $\lambda_{x}=\lambda_{y}$, the four cusp central curve of Figure 10 shrinks to a point at the origin: we recover the results of the preceding section: the origin $\rho=0$ is the map of the whole outer circle $\left(\mathrm{A}_{x}, \mathrm{~A}_{y}\right)$ which is an attractor in the $(x, y)$ plane. The multistable region shrinks to zero.

3.4. Hysteretic Behaviour. - In practice, only stable solutions are physically relevant, i.e. the part of the $(x, y)$ plane outside the external bifurcation curve in Figure 10. Everything that occurs inside that first bifurcation is irrelevant: there is no need to map these unphysical, unstable sheets. One should not forget however that the control parameter is $\left(\rho_{x}, \rho_{y}\right)$, not $(x, y)$ ! One should map the trajectories back into the $\rho$-plane, which is easily done using arguments of continulty. In that case one can locate the multistable regions: hysteretic jumps occur when one leaves these multistable regions, due to the disappearance of a local minimum. Each case must be considered separately.

The simplest situation is that of case $I I$ in Figure 10. Coming from $\rho_{y}=+\infty$, one reaches the spinodal limit in the lower half of the $\left(\rho_{x}, \rho_{y}\right)$ plane. It corresponds to a curve in the upper half of the $(x, y)$ plane. Multistability occurs in the shaded area. Whatever the direction of approach, the hysteretic jump occurs upon leaving that area. The coordinate $(x, y)$ jumps to another stable position pertaining to the same value of the control parameter $\left(\rho_{x}, \rho_{y}\right)$. This is llustrated in Figure 11a which sketches the one to one correspondence between the spinodal 
initial position $\mathbf{r}^{*}$ and the final position $\mathbf{r}_{\mathbf{f}}$ after the jump. $\mathbf{r}_{\mathbf{f}}$ lies in the stable part of the $(x$, $y$ ) plane, as it should. Note that in that plane the vicinity of $A_{x}$ is regular. In that regime the physics is essentially one dimensional, parametrized by the impact parameter $\rho_{y}$.

Case III is identical to case II: the secondary fold $\mathrm{B}_{x} \mathrm{~A}_{y}$ lies on the unstable sheet and is irrelevant. On the other hand the situation is somewhat different in case $I V$, as the bifurcation is primarily tangential. The spinodal limit corresponds to the four cusps curve $\mathrm{A}_{x} A_{y}$ of Figure 10 , whether that curve intersects or not the other bifurcation (the latter sits on another unstable sheet which is physically irrelevant: case IVa and IVb are identical). Once again discontinuous jumps occur only when one leaves the inside of that region. The vicinity of the cusps $A_{x}$ (in the "hard" direction) is simlar to that of case II. In the "soft" direction the cusp $A_{y}$ shows up in the curve of the corresponding final positions $\mathbf{r}_{\mathrm{f}}$, as displayed in Figure $11 \mathrm{~b}$ where $\mathrm{A}_{y}^{\prime}$ designates the final position corresponding to a jump at $A_{y}$. As could be surmised, the jumps are primarily in the soft direction $y$, with no spontaneous symmetry breaking (see Sect. 3.2).

As far as friction is concerned, the quantity of physical interest is the dissipated energy, i.e. the discontinuity in total energy $U$ due to the jump:

$$
\Delta U=U\left(\mathbf{r}_{\mathrm{f}}\right)-U\left(\mathbf{r}^{*}\right)
$$

An analytic expansion is possible only near the ends $\mathrm{A}_{x}$ elsewhere $\Delta U$ must be calculated numerically for each pinning potential $V(r)$. Here we only make a few qualitative comments on the dependence of $\Delta U$ on the elastic stiffness $\lambda_{x}$ and $\lambda_{y}$, which is markedly different in 1D and 2D. In one dimension the energy dissipation goes to $\infty$ as $\lambda$ goes to 0 . This somewhat paradoxical result is obvious in (3): for small $\lambda$ pinning can produce a huge elastic displacement $u=(x-\rho) \simeq 1 / \lambda$. The corresponding elastic energy is $\simeq u^{2} \times \lambda \simeq 1 / \lambda$ : it is basically released upon the jump. The reason for that is that there is no way to avoid the pinning center in 1D: trapping cannot be escaped. In contrast, a second dimension opens a new possibility: turning around the pinning center in order to avoid metastable states that ultimately build up large elastic energies: as a result $\Delta U$ goes through a maximum as a function of $\lambda$, returning to 0 when $\lambda$ gets smaller (as sketched in Fig. 12). Such an evolution can be studied quantitatively: here it is enough to look quickly at the limit $\lambda \rightarrow 0$. A glance at Figure 7 shows that such a limit always corresponds to case IV: the first bifurcation is always tangential, whether on the $x$ or on the $y$ axes. Consider for instance the $x$ axis: at bifurcation $x$ and $\rho_{x}$ are related by (9). They depend on the anisotropy $\lambda_{y} / \lambda_{x}$, but not on the absolute value of $\lambda_{x}$ and $\lambda_{y}$. Since $x$ lies necessarily within the range of the potential $V(r)$, it follows that $\rho_{x}$ also remains finite, with a logarithmic correction that we ignore, when $\lambda \rightarrow 0$ : the released energy upon jumping is of order $\lambda$ instead of $1 / \lambda$ !

In principle very small values of $\lambda$ and extreme anisotropy could conspire to produce cases II and III. Then the primary bifurcations are radial, corresponding to $\left(\lambda_{x}+V^{\prime \prime}\right)=0$. Equation (9) no longer holds: while $x$ is small, $\rho_{x}$ can become very large, implying a large elastic displacement $u$ - we recover the $1 \mathrm{D}$ situation in which $\Delta U$ grows when $\lambda$ become smaller. But such a case is artificial: for any reasonable anisotropy small stiffness means case IV!

\section{The Attractive Case}

The pinning potential $V$ is now negative: $V^{\prime}(r) / r$ is always positive as shown in Figure 13 . Tangential instabilities can no longer occur on the symmetry axes, while radial instabilities occur in pairs symmetric with respect to the origin. The relevant quantity is again the munimum value $V_{0}^{\prime \prime}$. Three cases may occur:

(i) $\left|V_{0}^{\prime \prime}\right| \leq \lambda_{y} \leq \lambda_{x}$ : the system is fully stable and there is no bifurcation at all - hence no hysteresis. 


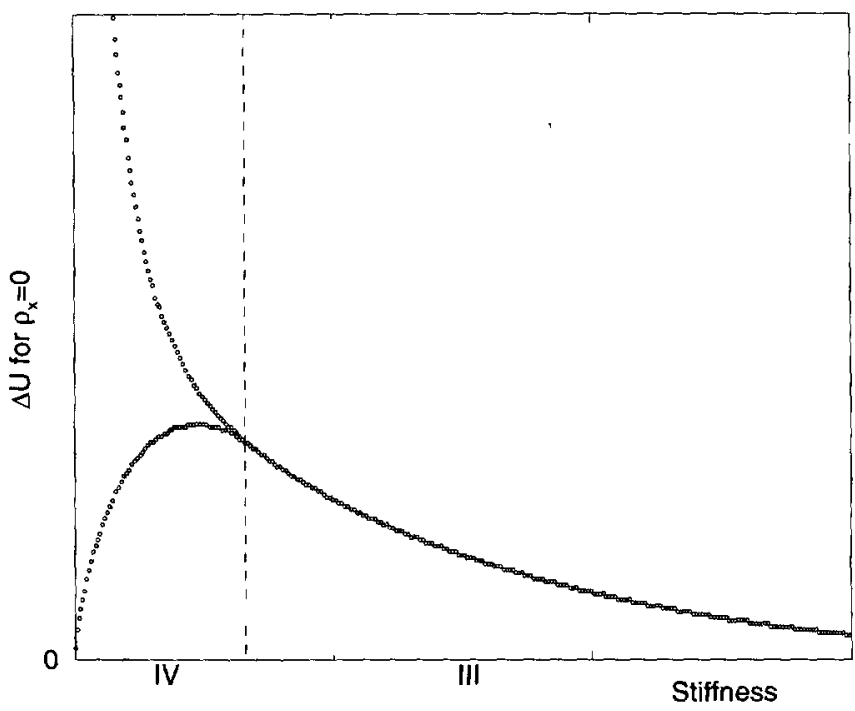

Fig. 12. - Dependence of the dissipated energy $\Delta U$ on stiffness $\lambda_{y} a^{2} /\left|V_{0}^{\prime \prime}\right|$ for $\rho_{x}=0$. The lower curve corresponds to the anisotropic case $\lambda_{x} / \lambda_{y}=1 / 2$. The upper curve corresponds to the $1 \mathrm{D}$ case. Zones III and IV are related to the corresponding regions of instability.

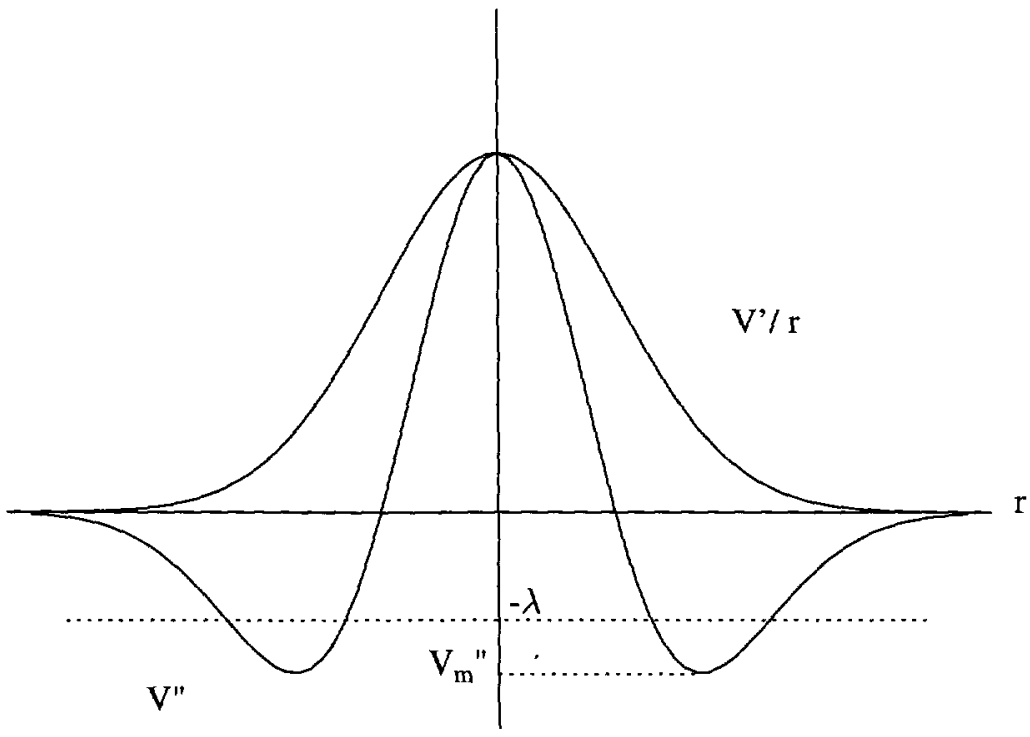

Fig. 13, - Geometrical construction of bifurcations on the symmetry axes in the attractive case.

(ii) $\lambda_{y} \leq \lambda_{x}<\left|V_{0}^{\prime \prime}\right|$ radial instabilities occur on both the $x$ and $y$ axes. In between, the bifurcation curves interpolate as shown in Figure 14 (the exact shape is obtained solving Eq. (14)). Regions 1 and 3 are locally stable, while region 2 corresponds to a saddle point of the total energy $U_{\text {eff }}$. When carried out in the $\left(\rho_{x}, \rho_{y}\right)$ plane (using (8)), the two curves of the $(x, y)$ plane interchange, as shown in Figure 14b. This is most easily seen in the 


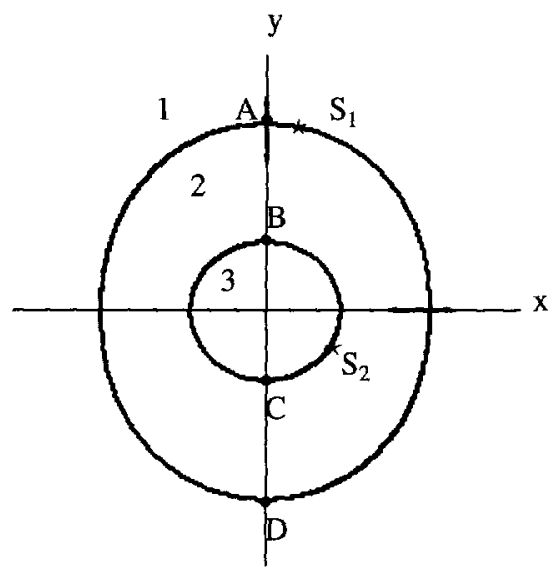

(a)

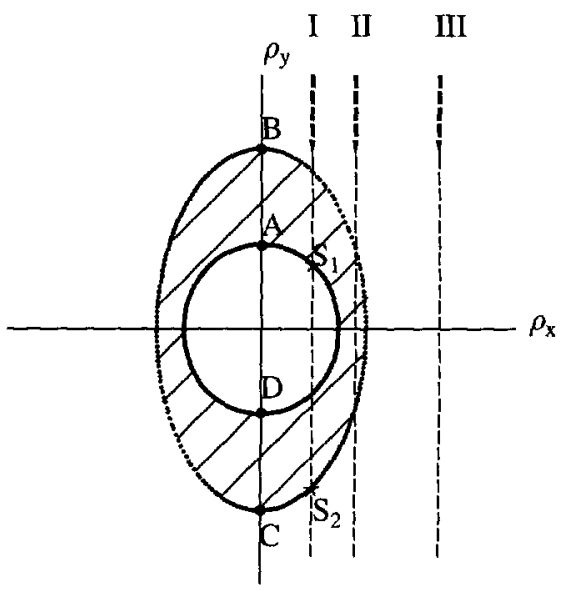

(b)

Fig. 14. - Bifurcation diagram in the attractive case when $\lambda_{x}<\lambda_{y}<\left|V_{0}^{\prime \prime}\right|$. The hatched area is that multistability. I, II, III represent three different sweepmg paths at constant $\rho_{x}$. Spinodal jumps occur at $S_{1}$ and $S_{2}$ for path $I$ only.

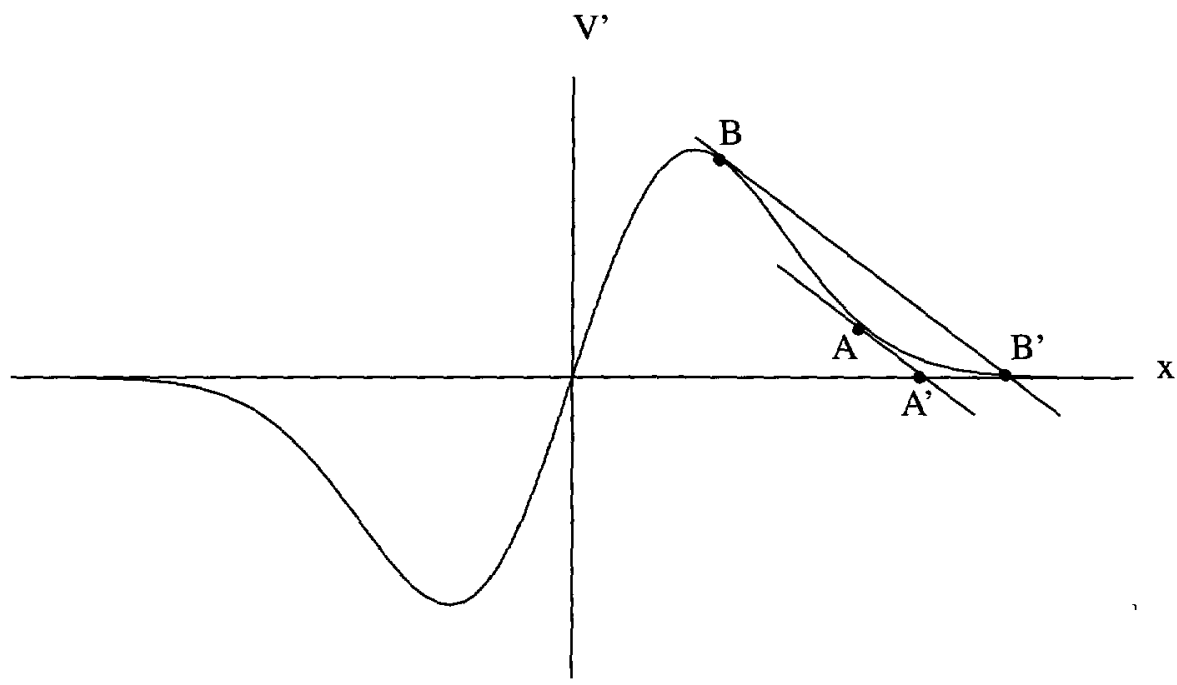

Fig. 15. - Geometrical construction of $\rho_{x}$ for a radial instability along the $x$ axis in the attractive case. The labelling of points is the same as in Figure 14.

graphical construction of Figure 15, which yields $\rho_{x}$ given $x$ at the bifurcation point. Such an interchange is crucial, as it means that the hatched area in Figure $14 \mathrm{~b}$ is multistable. As usual the argument relies on continuity as the representative point is brought from infinity in the $(x$, $y$ ) plane. Assume for instance that we come along the $y$-axis downward: we meet instability at point $A$ and we recover another stable branch at $B$. In between $\rho_{y}(y)$ is reentrant: an unstable branch goes backward from $A$ to $B$. The same situation occurs in the lower half of the 


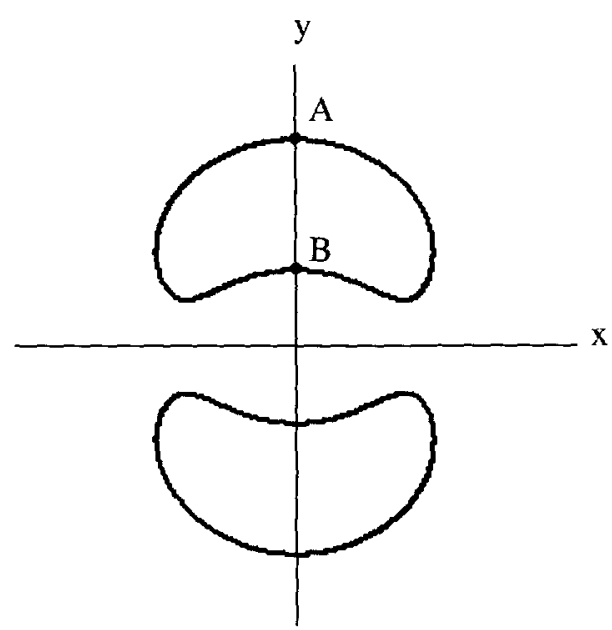

(a)

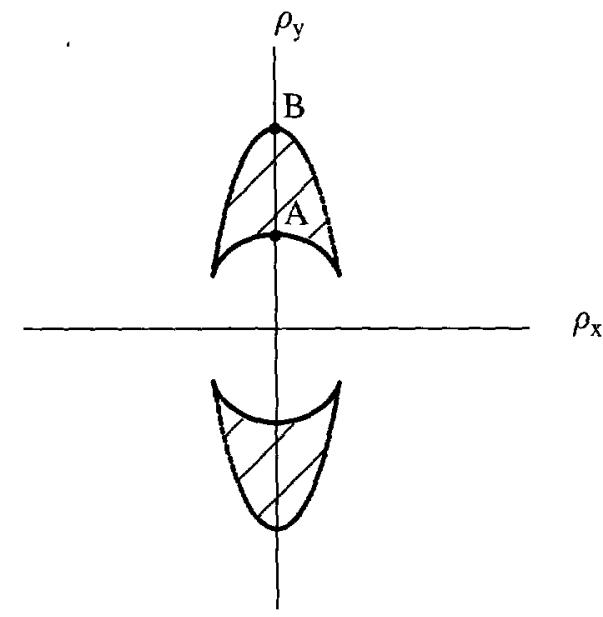

(b)

Fig. 16. - Bifurcations diagram of the attractive case when $\lambda_{y}<\left|V_{\mathrm{m}}^{\prime \prime}\right|<\lambda_{x}$. The shaded areas are multistable.

figure: the reentrant part goes from $\mathrm{C}$ to $\mathrm{D}$.

Multistability is responsible for hysteresis. Assume for instance that $\rho$ is swept downward in the $y$-direction, with a constant value of the "impact parameter" $\rho_{x}$. As seen in Figure 14b there are three possibilities:

- In case III there is no instability whatsoever: the actual position $\mathbf{r}$ varies continuously as $\rho$ is varied.

- In case I spinodal discontinuous jumps occur when one meets the first instability, i.e. when one leaves the multistable region - at points $S_{1}$ and $S_{2}$ in Figure 14. There are two hysteresis cycles, in contrast to the repulsive case.

- In case II the answer is more subtle. One never meets the inner curve AD of Figure $14 \mathrm{~b}$, which according to Figure 14a provides the first instability in the $(x, y)$ plane. It follows that the evolution is perfectly continuous: in the $(x, y)$ plane one goes around the dangerous outer curve. The trajectory remains in the stable region and there is no hysteresis. We described a similar situation in the isotropic repulsive problem.

In the isotropic limit $\lambda_{x}=\lambda_{y}=\lambda$, the bifurcation curves of Figure 16 become circles. Full rotational symmetry is restored and the bifurcations are everywhere strictly radial.

(iii) $\lambda_{y}<\left|V_{0}^{\prime \prime}\right|<\lambda_{x}$ : there is no instability whatsoever along the $x$ axis. The bifurcation curve in the $(x, y)$ plane, given by (14), should then split into two disjoint loops as shown in Figure 16a (this is the only way to connect smoothly the two radial bifurcations along the $y$ axis). In the $(x, y)$ plane these curves are regular. The corresponding map in the $\left(\rho_{x}, \rho_{y}\right)$ plane is shown in Figure 16b: once again the shaded area is multistable, ylelding hysteresis whenever the sweeping trajectory crosses it. In that plane the bifurcation curve is analytic on the $y$ axis, as shown in the Appendix. On the other hand it should display cusps at the extremities, where the multistability disappears. Such a behaviour is a standard feature of "fold catastrophes" as a function of the control parameter. 
Since symmetry is here useless we need a direct proof. Assume that the bifurcation curve is parametrized by a variable $s$. A cusp occurs if one has simultaneously:

$$
\frac{\mathrm{d} \rho_{x}}{\mathrm{~d} s}=\frac{\mathrm{d} \rho_{y}}{\mathrm{~d} s}=0
$$

(choosing $s=0$ at the cusp, we expand the derivatives as $\mathrm{d} \rho_{2} / \mathrm{d} s=a_{\imath} s+b_{2} s^{2}+\ldots$. The ratio $a_{y} / a_{x}$ fixes the slope of the cusp, the $b_{2}$ control the approach to that slope; the components of $\rho$ parallel and perpendicular to the cusp behave respectively as $s^{2}$ and $s^{3}$ ). In Figure $16 \mathrm{~b}$ we may take the coordinate $y$ as the parameter. Returning to Cartesian coordinates (8) becomes

$$
\rho_{x}=x+\frac{V_{x}^{\prime}}{\lambda_{x}} \quad, \quad \rho_{y}=y+\frac{V_{y}^{\prime}}{\lambda_{y}}
$$

It follows that

$$
\begin{aligned}
& \frac{\mathrm{d} \rho_{x}}{\mathrm{~d} y}=\frac{V_{x y}^{\prime \prime}}{\lambda_{x}}+\left(1+\frac{V_{x x}^{\prime \prime}}{\lambda_{x}}\right) \frac{\mathrm{d} x}{\mathrm{~d} y} \\
& \frac{\mathrm{d} \rho_{y}}{\mathrm{~d} y}=\frac{V_{y y}^{\prime \prime}}{\lambda_{y}}+\left(1+\frac{V_{x y}^{\prime \prime}}{\lambda_{y}}\right) \frac{\mathrm{d} x}{\mathrm{~d} y}
\end{aligned}
$$

where $\mathrm{d} x / \mathrm{d} y$ fixes the slope of the bifurcation curve $\mathrm{C}$ in the $(x, y)$ plane. The result (19) should be compared to the equations that give the components $(\xi, \eta)$ of the soft mode at any point of $\mathrm{C}$ :

$$
\begin{aligned}
& \left(\lambda_{x}+V_{x x}^{\prime \prime}\right) \xi+V_{x y}^{\prime \prime} \eta=0 \\
& V_{x y}^{\prime \prime} \xi+\left(\lambda_{y}+V_{y y}^{\prime \prime}\right) \eta=0
\end{aligned}
$$

A cusp occurs of $\xi / \eta=\mathrm{d} x / \mathrm{d} y$, i.e. if the soft mode is tangent to the bifurcation curve $\mathrm{C}$ (as found by symmetry in the repulsive case). That occurs somewhere in Figure 16: it is obvious when $\lambda_{x}$ is infinite (the soft mode is polarized along the $y$-direction). It remains true by continuity at finite $\lambda_{x}$. Hence the cusps of Figure $16 \mathrm{~b}$.

\section{Conclusion}

The scenario for first order transitions and accompanying hysteresis is well-known in a onedimensional geometry. In this paper we have shown that the extension to a two-dimensional case is non trivial. The idea of multistability and hysteresss remains valid, but the variety of scenarios is much larger. Crudely speaking, the existence of a second dimension opens the possibility of "escape", away from bifurcations, leading to three distinct kinds of behaviour for our system. Both regions of multistability and dissipated energy are thereby reduced by this escape. Such a behaviour is clearly demonstrated in the isotropic repulsive case, in which the whole hysteretic region shrinks to zero (the representative point turns around the conical point of $U_{\text {eff }}$ ). As the anisotropy grows, the behaviour evolves toward the $1 \mathrm{D}$ case, as expected. We provide a qualitative description of that evolution.

Note that the second dimension changes crudely the variations of the dissipated energy versus stiffness, and that a maximum of dissipated energy appears in the regime of "escape" in the anısotropic case. In the attractive case, this regime of "escape" is not possible and the dissipated energy depends only on the distance to the center of pinning. 
Our work was originally motivated by a recent model of solid friction, based on hysteresis of local pinning centers on sliding surfaces [1]. Such an approach is not without interest especially for the study of AFM's experiments [4]. The extension to an additional vertical dimension [5] could, in the same way, allow a better understanding of the influence of the normal charge on the friction coefficient. Note also that the dynamic of such systems can be very complex [6]. In this paper, we used the mentioned model of solid friction in order to present our results. But clearly the issue is much more general: it bears on the nature of phase transitions in a multidimensional space, an important problem in many different contexts. Going beyond qualitative statements would be irrelevant, as quantitative developments depend on the problem at hand. As it stands the present paper is mostly indicative.

\section{Acknowledgments}

This work was initiated by Christiane Carolı at GPS-Jussieu. The authors wish to express their gratitude for her advice and encouragement. A. Tanguy is grateful for the facilities offered at LPMMH for the completion of this work.

\section{Appendix A}

In order to substantiate our qualitative discussion, we use the choice (12). We set

$$
\lambda_{x}=\left|V_{0}^{\prime \prime}\right|\left(1+\epsilon_{x}\right) \quad, \quad \lambda_{y}=\left|V_{0}^{\prime \prime}\right|\left(1+\epsilon_{y}\right)
$$

with the constraint $-1<\epsilon_{y}<\epsilon_{y}$. Instabilities appear if $\epsilon_{x}$ and/or $\epsilon_{y}$ are negative. Using the appropriate units for the energy $U$ and for the lengths $r$, we may set $\left|V_{0}^{\prime \prime}\right|=\alpha=1$. Equations (8) then reduce to

$$
\rho_{x}=\frac{x}{1+\epsilon_{x}}\left(\epsilon_{x}+r^{2}\right) \quad, \quad \rho_{y}=\frac{y}{1+\epsilon_{y}}\left(\epsilon_{y}+r^{2}\right)
$$

while the determinant $\Delta$ is

$$
\Delta=3 r^{2}+\epsilon_{x}\left(x^{2}+3 y^{2}\right)+\epsilon_{y}\left(y^{2}+3 x^{2}\right)+\epsilon_{x} \epsilon_{y}
$$

Expansions in the vicinity of the $x$ axis and $y$ axis are then straightforward. We find the following.

\section{Vicinity of $A_{x}$}

The bifurcation is parametrized by $y$. The other coordinates are

$$
\begin{aligned}
x & =\sqrt{-\epsilon_{y}}\left(1-\frac{y^{2}}{2 \epsilon_{y}}-\frac{3 \epsilon_{x}-5 \epsilon_{y}}{3 \epsilon_{y}-\epsilon_{x}}\right) \\
\overline{\rho_{x}} & \equiv\left(1+\epsilon_{x}\right) \rho_{x}=\sqrt{-\epsilon_{y}}\left(\epsilon_{x}-\epsilon_{y}\right)\left(1+\frac{3 y^{2}}{2 \epsilon_{y}}\right) \\
\overline{\rho_{y}} & \equiv\left(1+\epsilon_{y}\right) \rho_{y}=2 \frac{\left(\epsilon_{x}-\epsilon_{y}\right)}{3 \epsilon_{y}-\epsilon_{x}} y^{3}
\end{aligned}
$$

$\mathrm{A}_{x}$ is a cusp in the $\left(\rho_{x}, \rho_{y}\right)$ plane $\left(\rho_{y} \sim \Delta \rho_{x}{ }^{\frac{3}{2}}\right)$. The signs are those shown in Figure 10. 


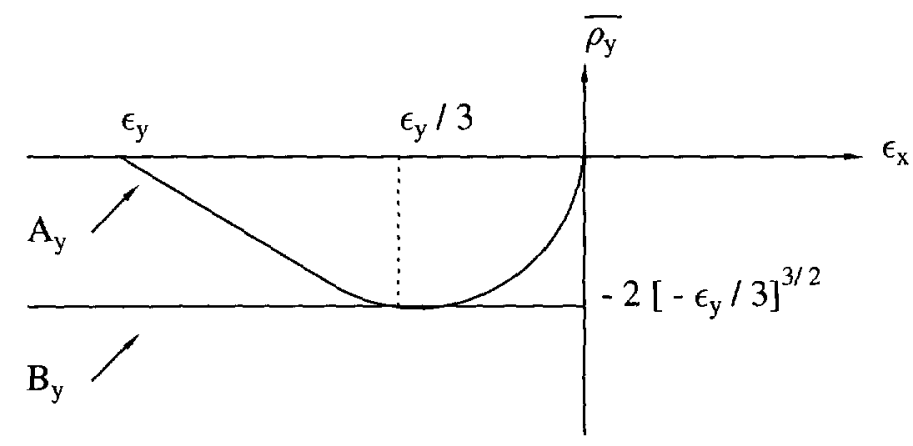

Fig. 17. - Ordinates of the cusps on the $\overline{\rho_{y}}$ axis: as explained in the text $A_{y}$ remains closer to the origin than $B_{y}$.

\section{Vicinity of $\mathrm{B}_{y}$}

$$
\begin{aligned}
y & =\sqrt{\frac{-\epsilon_{y}}{3}}\left(1-\frac{3 x^{2}}{2 \epsilon_{y}} \frac{\epsilon_{x}+\epsilon_{y}}{\epsilon_{y}-3 \epsilon_{x}}\right) \\
\overline{\rho_{x}} & =x\left(\frac{3 \epsilon_{x}-\epsilon_{y}}{3}\right) \\
\overline{\rho_{y}} & =-2\left(\frac{-\epsilon_{y}}{3}\right)^{3 / 2}\left(1+\frac{3 x^{2}}{2 \epsilon_{y}}\right)
\end{aligned}
$$

The bifurcation curve is regular in the $\left(\rho_{x}, \rho_{y}\right)$ plane.

\section{Vicinity of $\mathrm{A}_{y}$ and $\mathrm{B}_{x}$}

It is obtained by interchanging $x$ and $y$ in $A_{x}$ and $B_{y}$.

For $A_{y}$, for instance,

$$
\begin{aligned}
y & =\sqrt{-\epsilon_{x}}\left(1-\frac{x^{2}}{2 \epsilon_{x}}-\frac{3 \epsilon_{y}-5 \epsilon_{x}}{3 \epsilon_{x}-\epsilon_{y}}\right) \\
\overline{\rho_{y}} & =\sqrt{-\epsilon_{x}}\left(\epsilon_{y}-\epsilon_{x}\right)\left(1+\frac{3 x^{2}}{2 \epsilon_{x}}\right) \\
\overline{\rho_{x}} & =2 \frac{\left(\epsilon_{y}-\epsilon_{x}\right)}{3 \epsilon_{x}-\epsilon_{y}} x^{3}
\end{aligned}
$$

From these results the following conclusions ensue

- The transition between regimes III and IV (coalescence of $A_{y}$ and $B_{y}$ ) occurs for $\epsilon_{y}=3 \epsilon_{x}$. As a function of $\epsilon_{x}$ the corresponding coordinates $\overline{\rho_{y}}$ on the symmetry axis behave as shown in Figure 17: $\mathrm{A}_{y}$ touches $\mathrm{B}_{y}$ but it does not cross it.

- Near $\mathrm{A}_{y}$ and $\mathrm{B}_{y}$ the coordinate $\overline{\rho_{x}}$ changes sign when $\epsilon_{y}=3 \epsilon_{x}$, thereby marking the interchange of the two branches in the $\left(\rho_{x}, \rho_{y}\right)$ plane: see Figure 10.

- In the $(x, y)$ plane, the curvature near $\mathrm{B}_{y}$ changes sign when $\epsilon_{x}=-\epsilon_{y}$ : it acquires an outward convexity. Near the III-IV transition, $\epsilon_{y}=3 \epsilon_{x}$, the curvatures of $A_{y}$ and $\mathrm{B}_{y}$ exchange, as shown in Figure 18. The corresponding bifurcation is usually termed "transcritical". 


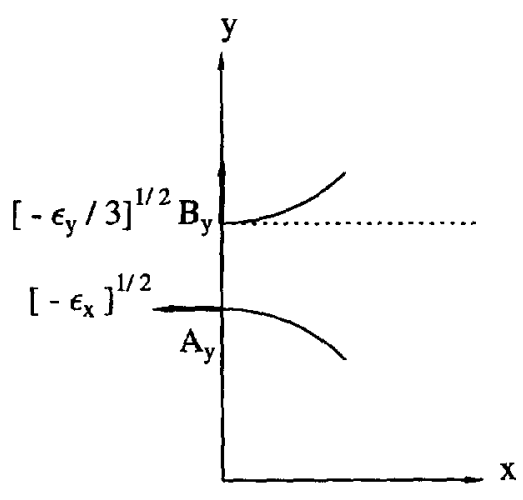

$$
\epsilon_{\mathrm{y}}<3 \epsilon_{\mathrm{x}}
$$

III

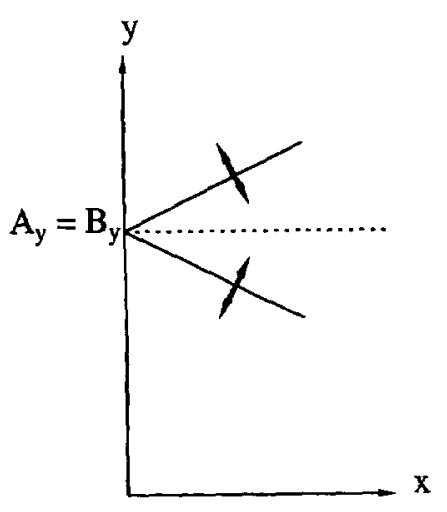

$$
\epsilon_{\mathrm{y}}=3 \epsilon_{\mathrm{x}}
$$

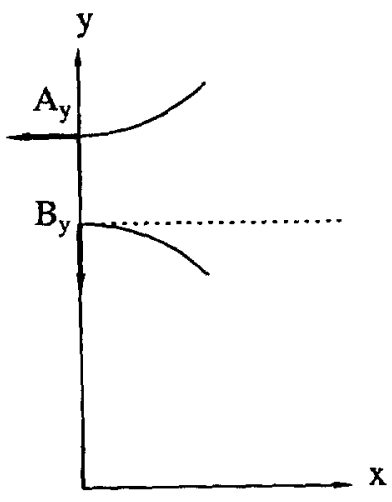

$$
\epsilon_{\mathrm{y}}>3 \epsilon_{\mathrm{x}}
$$

IV

Fig. 18. - The vicinity of the III-IV transition.

The shrinking to zero of $\mathrm{A}_{x}$ and $\mathrm{A}_{y}$ as the isotrop1c limit $\epsilon_{x}=\epsilon_{y}$ is approached is clearly apparent. We also find that the passage from regimes IVa to IVb (Fig. 10) occurs at $\epsilon_{x}=3 \epsilon_{y} / 4$. All these results are accurate near the minimum $V_{0}^{\prime \prime}$ in Figure 7 . They become shape dependent away from that minimum.

The occurrence of cusps in the $\left(\rho_{x}, \rho_{y}\right)$ plane is a general feature which does not depend on our particular choice of $V$. Consider for instance the vicinity of the $x$-axis: radial instabilities correspond to $\left(V^{\prime \prime}+\lambda\right)=0$. When carried out in (8) such a root does not create any singularity: $\rho_{y}$ and $\rho_{x}$ are respectively of order $y$ and $y^{2}$ by symmetry and the bifurcation curve in the $\rho$ plane is regular. In contrast a tangential instability corresponds to $\left(\left(V^{\prime} / r\right)+\lambda_{y}\right)=0:(8)$ then implies that $\rho_{y}$ is of order $y^{3}, \rho_{x}$ remanning of order $y^{2}$, hence a cusp.

\section{References}

[1] Caroli C. and Nozières P., in The Physics of sliding friction, B. N.J. Persson Ed. (1995).

[2] Landau I., Phys. Z. Sowjet. 11 (1937) 26; II. Phys. Z. Sowjet. 11 (1937) 545.

[3] Lu, Singularity theory and an Introduction to Catastrophe theory (Springer Verlag ed., 1976) chap. 4.

[4] Gyalog T., Bammerlin M., Lüthi R., Meyer E. and Thomas H., Europhys. Lett. 31 (1995) 269.

[5] Preprint.

[6] Hale J. and Koçak H., Dynamics and bifurcations (Sprınger Verlag ed., 1991). 